appointments, which may underestimate lower EDSS. Future clinics could combine both modalities.

\section{HEADACHES OF RAISED INTRACRANIAL PRESSURE AS A PRESENTING FEATURE OF MALIGNANT INFILTRATION IN THE CAUDA EQUINA}

${ }^{1}$ Matthew Silsby, ${ }^{2}$ Andrew Martin, ${ }^{3}$ Winny Varikatt, ${ }^{1}$ Steve Vucic, ${ }^{1}$ Victor SC Fung, ${ }^{1}$ Parvathi Menon. 'Neurology, Westmead Hospital, Westmead, NSW, Australia; ${ }^{2}$ Neurology, Blacktown Hospital, Blacktown, NSW, Australia; ${ }^{3}$ Anatomical Pathology, Westmead Hospital, Westmead, NSW, Australia

\subsection{6/bmjno-2021-ANZAN.73}

Introduction Raised intracranial pressure (ICP) headache is rarely caused by spinal cord lesions. We present two patients with rare presentations of uncommon malignancies surrounding the cauda equina resulting in raised ICP headache.

Cases Patient 1, a 54-year-old man, presented with two months of headache, blurred vision and $9 \mathrm{~kg}$ weight loss. Fundoscopy revealed papilloedema. Shortly after presentation he developed lower limb weakness with absent tendon reflexes. MRI brain was unremarkable. MRI spine showed diffuse nerve root thickening and effacement of the CSF spaces around the cauda equina. Patient 2, a 51-year-old man, presented with four months of headache and blurred vision, progressive lower limb weakness and $20 \mathrm{~kg}$ weight loss over 12 months. MRI brain showed two small lesions insufficient to cause raised ICP. MRI spine showed extensive lower spinal cord infiltration with diffuse thickening of the cauda equina. Pathological assessments revealed malignant histiocytosis in patient 1 and primary leptomeningeal gliomatosis in patient 2 .

Discussion These cases demonstrate the rare presentation of raised ICP headache resulting from spinal lesions. In both cases the original presentation with raised ICP headache and absent lower limb signs led to diagnostic delay. Malignant lesions in the spinal column can increase ICP, though the mechanism is debated. These cases emphasise the importance of a broader search for the aetiology of raised ICP headache in the absence of a causative lesion on primary brain imaging. Further, these cases highlight rare presentations of two uncommon pathological entities.

\section{CEFTRIAXONE THERAPY FOR ADULT ALEXANDER DISEASE: REPORT OF 2 CASES}

${ }^{1}$ Natalie C Palavra, ${ }^{2,3}$ Omar Ahmad. 'Department of Neurology, Royal North Shore Hospital, Northern Sydney Local Health District, St Leonards, NSW, Australia; ${ }^{2}$ Department of Neurology, Sydney Adventist Hospital, Wahroonga, NSW, Australia; ${ }^{3}$ Department of Neurology, Hornsby Ku-Ring-Gai Hospital, Northern Sydney Local Health District, Hornsby, NSW, Australia

\subsection{6/bmjno-2021-ANZAN.74}

Objectives Adult onset Alexander Disease (AxD) is a rare leukodystrophy for which there is currently no treatment. Ceftriaxone has been proposed as a potential treatment for AxD. Here we report the clinical outcome of an extended course of intravenous cyclical ceftriaxone therapy in two patients with AxD.
Methods Case 1 is a 64 year old female with a five year history of progressive gait disturbance, bulbar palsy and horizontal diplopia. Case 2 is an 80 year old male with a two-year history of bulbar palsy and gait disturbance. Both cases were confirmed to have $\mathrm{AxD}$ and received intravenous ceftriaxone $2 \mathrm{~g}$ daily for three weeks per month during the initial four months, then for 15 days monthly thereafter. Patients were assessed at baseline and approximately 4-month intervals using the Kurtzke Expanded Disability Status Scale, Modified Ranking Scale, and neurological examination.

Results Both cases displayed functional decline on ceftriaxone therapy, as assessed by outcome measures of disability. Progression on neuroimaging was also observed on MRI brain for both patients.

Conclusions These results suggest that ceftriaxone for AxD may not prevent functional decline. Caution should be applied before suggesting ceftriaxone for the management of $\mathrm{AxD}$.

\section{GADOLINIUM ENCEPHALOPATHY PRESENTING AS STATUS EPILEPTICUS FOLLOWING INTRATHECAL INJECTION}

${ }^{1}$ Emily Sutherland, ${ }^{1} J$ onathan Baird-Gunning, 'Laura Rudaks, ${ }^{1}$ Natalie Palavra, ${ }^{1,2}$ Michal Lubomski, 'Martin Krause. 'Department of Neurology, Royal North Shore Hospital, St Leonards, NSW, Australia; ${ }^{2}$ Neurological Sciences, Prince of Wales Hospital, Randwick, NSW, Australia

\subsection{6/bmino-2021-ANZAN.75}

Objectives Intra-thecal gadolinium is an alterative contrast agent for computed tomography myelograms. Uncommonly it can cause gadolinium encephalopathy which can present with an array of neurological signs and symptoms.

Methods Review of a case shared by interventional radiology, emergency, neurology and intensive care. This case describes the symptomatology, investigations, and treatment for gadolinium encephalopathy, alongside recommended dosages for intrathecal administration.

Results An 85 year-old female with an allergy to intra-venous iodine presented for a computed tomography myelogram for investigation of bilateral lower limb pain. During the myelogram $8 \mathrm{~mL}$ of intrathecal gadolinium (Gadovist 1.0) equivalent to $8 \mathrm{mmol}$ of gadobuterol was injected in light of the iodine allergy. Ten minutes after completion of the procedure the patient had abrupt onset pelvic pain, nausea, and bilateral lower limb paraesthesiae, which was treated as an allergic reaction with an anti-emetic, analgesia, and steroids. Rapid deterioration followed with extreme agitation and subsequent convulsive status epilepticus. After intubation and treatment with anti-epileptic medication an EEG showed persistent nonconvulsive status epilepticus. Intra-venous steroids were introduced, alongside two more anti-epileptics. She was extubated on day 7 , and EEG normalised by day 24. The patient was discharged to a rehabilitation hospital with moderate residual cognitive impairments.

Conclusion This case outlines a recognised but infrequently reported response to intrathecal gadolinium. Guidelines for safe intrathecal injection are yet to be identified, as are specific treatment options. 